\title{
Photoemission von $\mathrm{Gd}, \mathrm{Tb}$ und den metallreichen Chloriden $\mathrm{Gd}_{2} \mathrm{Cl}_{3}$ und $\mathrm{Tb}_{2} \mathrm{Cl}_{3}$
}

\author{
G. Ebbinghaus, A. Simon und A. Griffith \\ Max-Planck-Institut für Festkörperforschung, Stuttgart \\ Z. Naturforsch. 37a, 564-567(1982); received March 26, 1982 \\ Photoemission of $\mathrm{Gd}, \mathrm{Tb}$ and the metal-rich chlorides $\mathrm{Gd}_{2} \mathrm{Cl}_{3}$ and $\mathrm{Tb}_{2} \mathrm{Ch}_{3}$
}

The photoelectron spectra ( $\mathrm{AlK} \alpha, \mathrm{HeII}, \mathrm{HeI}$ ) of $\mathrm{Gd}, \mathrm{Tb}, \mathrm{Gd}_{2} \mathrm{Cl}_{3}$ and $\mathrm{Tb}_{2} \mathrm{Cl}_{3}$ are measured and discussed. They clearly indicate $4 \mathrm{f}^{7}$ - and $4 \mathrm{f}^{8}$-configuration for the sesquihalides of Gd and $\mathrm{Tb}$, respectively. In the case of $\mathrm{Gd}_{2} \mathrm{Cl}_{3}$ a band gap of approximately $1 \mathrm{eV}$ is estimated.

\section{Einleitung}

In den Verbindungen $\mathrm{Gd}_{2} \mathrm{Cl}_{3}[1-3]$ und $\mathrm{Tb}_{2} \mathrm{Cl}_{3}$ $[3,4]$ liegen die Seltenerdmetalle in ungewöhnlich niedriger Oxidationsstufe vor. Die Kristallstrukturen bieten eine Erklärung: Die am Metallatom verbliebenen Valenzelektronen bauen Metall-Metall-Bindungen auf. Es treten unendliche Ketten kondensierter $\mathrm{Ln}_{6}$-Oktaeder auf [5], die nach Art des $\mathrm{M}_{6} \mathrm{X}_{8}$ Clusters von Cl-Atomen umgeben sind. Die Kristallstruktur legt besonders zwei Fragen nahe: (a) Sind die Verbindungen eindimensionale Metalle? (b) Wie ist die chemische Bindung beschreibbar?

\section{Experimentelle Angaben}

Die Messungen der Photoemission sind in einem Leybold-Heraeus-LHS-10-ESCA-Spektrometer mit HeI-, HeII- und AlK $\alpha$-Strahlung ohne Monochromatisierung durchgeführt worden. Bei den gewählten Anregungsarten ( $h v=21,2,40,8$ und $1486,6 \mathrm{eV}$ ) beträgt die instrumentelle Energieauflösung 0,1, 0,2 bzw. 1,2 eV. Nach dem Ausheizen ist der Druck im Analysatorraum kleiner als $1 \cdot 10^{-10} \mathrm{mbar}$. Während der Messungen mit der fensterlosen KaltkathodenGasentladungslampe steigt der Druck auf 3 . $10^{-9} \mathrm{mbar}$ (He, $6 \mathrm{~N}$-Reinheit) an. Der Basisdruck im Präparationsraum beträgt etwa $5 \cdot 10^{-10}$ mbar.

Aus kompakten Stücken der Metalle (Reinheit 99,9\%, Fa. Kelpin) werden im Handschuhkasten Scheiben passender Größe geschnitten. Die Proben

Sonderdruckanforderungen an Prof. Dr. A. Simon, MaxPlanck-Institut für Festkörperforschung, Heisenbergstr. 1, D-7000 Stuttgart 80. werden unter getrocknetem Argon ins Spektrometer gebracht und nach dem Ausheizen mit einer Diamantfeile gereinigt. Auch unter den oben angegebenen Vakuumbedingungen beobachtet man innerhalb weniger Minuten eine Veränderung der UV-Spektren durch Kontamination. Deshalb werden die HeI- bzw. HeII-Spektren innerhalb von einer bzw. fünf Minuten gemessen. Mehrere an frischen Probenoberflächen erhaltene Spektren werden addiert. Obwohl sich die Spektren bei Anregung mit Röntgen-Strahlen nach 12 Stunden nicht sichtbar verändern, werden die Proben auch hier (jeweils nach 3 Stunden) durch Abfeilen im UHV gereinigt.

Alle Handhabungen der Verbindungen erfolgen wegen ihrer Feuchtigkeitsempfindlichkeit unter getrocknetem Argon. Die $\mathrm{Ln}_{2} \mathrm{Cl}_{3}$-Proben werden in mehreren Schritten hergestellt: Das aus der Reaktion von Metall und Trihalogenid gewonnene feinkristalline Produkt [3] wird zu Tabletten gepreßt, die in verschweißten Tantaltiegeln bei ca. $500{ }^{\circ} \mathrm{C}$ mehrere Tage getempert werden. Die Tabletten werden anschließend in eine passende Vertiefung des Probenhalters gelegt und zum Einbau des Halters zunächst mit einem Teflon-gedichteten Deckel vor Luftzutritt geschützt. Im Vakuum des Spektrometers wird der Deckel entfernt, die Probe bei $200{ }^{\circ} \mathrm{C}$ ausgeheizt und zur Messung oberflächlich mit einem gehärteten Stahlstempel abgerieben.

Wird die $\mathrm{Gd}_{2} \mathrm{Cl}_{3}$-Probe etwa 12 Stunden unter gleichzeitigem Ar-Ionenbeschuß bei $p=10^{-5} \mathrm{mbar}$ Ar-Druck auf $600{ }^{\circ} \mathrm{C}$ erhitzt, so wird anschließend ein verändertes Spektrum gemessen. Das XPS-Spektrum spricht für die Bildung von $\mathrm{GdCl}[4,6]$, das sich unter rein thermischen Bedingungen nur unter Schwierigkeiten aus $\mathrm{Gd}_{2} \mathrm{Cl}_{3}$ erhalten läßt. 
Tab. 1. Bindungsenergien [eV] für $\mathrm{Gd}, \mathrm{Tb}, \mathrm{Gd}_{2} \mathrm{Cl}_{3},,, \mathrm{GdCl}^{\prime \prime}$ und $\mathrm{Tb}_{2} \mathrm{Cl}_{3}$. Die Werte sind auf das Fermi-Niveau $\left(E_{\mathrm{F}}=0 \mathrm{eV}\right)$ bezogen.

\begin{tabular}{|c|c|c|c|c|c|c|c|}
\hline \multicolumn{8}{|l|}{ Niveau } \\
\hline Probe & $\mathrm{M}_{3} \mathrm{~d}_{3 / 2}$ & $\mathrm{M}_{3 / 2}$ & M5p $p_{1,2}$ & M5р $3 / 2$ & M4f & $\mathrm{Cl} 3 \mathrm{~s}$ & $\mathrm{Cl} 3 \mathrm{p}$ \\
\hline $\mathrm{Gd}_{2} \mathrm{Cl}_{3}$ & $1222,0(10)$ & $1190,2(5)$ & \multicolumn{2}{|c|}{$31-22,5$} & $10,0(2)$ & $18,4(5)$ & $7,4(2)$ \\
\hline$[,, \mathrm{GdCl} "]$ & $1220,2(5)$ & $1188,3(5)$ & $31-26$ & $25-20$ & $9,7(2)$ & $18,0(5)$ & $7,1(2)$ \\
\hline & $1218,7(5)$ & $1186,3(5)$ & $28,5-24$ & $24-19,5$ & $8,3(2)$ & - & - \\
\hline $\mathrm{Tb}_{2} \mathrm{Cl}_{3}$ & $1278(1)^{*}$ & $1244(1)^{*}$ & \multirow{2}{*}{\multicolumn{2}{|c|}{$32-21$}} & Multiplett & $17,6(5)$ & $7,4(2)$ \\
\hline & $1275(1)^{*}$ & $1241(1)^{*}$ & & & Multiplett & - & - \\
\hline Gd [7] & $1221,9(8)$ & $1189,6(8)$ & & & $8,6(1)$ & - & - \\
\hline $\mathrm{Tb}[7]$ & $1278,8(8)$ & $1243,2(8)$ & & & $\begin{array}{l}7,8\left[{ }^{8} \mathrm{~S}\right] \\
2,6\end{array}$ & - & - \\
\hline $\mathrm{Tb}[8]$ & $1275,0(2)$ & $1239,1(2)$ & $28,7(2)$ & $22,6(2)$ & & - & - \\
\hline
\end{tabular}

* Die Multiplettstrukturen für Tb 3d konnten nicht aufgelöst werden; die Peak-Schwerpunkte sind angegeben.

\section{Ergebnisse und Diskussion}

In der Tabelle werden die beobachteten Bindungsenergien aufgeführt. Die gemessenen Werte für $\mathrm{Gd}$ und $\mathrm{Tb}$ sind etwa 2 bis $3 \mathrm{eV}$ kleiner als die von Kowalczyk [7] berichteten; bei $\mathrm{Tb}$ entsprechen die eigenen Meßwerte den von Padalia et al. [8] angegebenen. Parallel zur Oxidationsstufe des Metalls findet man chemische Verschiebungen der Rumpfelektronen-Niveaus, die für das $\mathrm{Gd} 3 \mathrm{~d}_{5 / 2}$-Niveau $2,0 \mathrm{eV}$ für " $\mathrm{GdCl}$ “ und $3,9 \mathrm{eV}$ für $\mathrm{Gd}_{2} \mathrm{Cl}_{3}$ betragen. Die Spin-Bahn-Aufspaltung des Gd 3d-Dubletts ist für „ $\mathrm{GdCl}$ “ und $\mathrm{Gd}_{2} \mathrm{Cl}_{3}$ geringfügig erniedrigt; allgemein wird bei Verbindungsbildung eine Abnahme der Spin-Bahn-Aufspaltung nur für schwach gebundene Rumpfniveaus beobachtet [9, 10].

Das Aussehen der Spektren hängt wesentlich von der anregenden Strahlung ab. Abbildung 1 illustriert am Beispiel der Spektren von Gd, daß bei verschiedenen Photonenenergien aufgrund der wechselnden Anregungswahrscheinlichkeiten für Elektronen mit unterschiedlichen Wellenfunktionen erhebliche Intensitätsunterschiede auftreten. Ferner werden die Spektren durch unterschiedliche Empfindlichkeit auf oberflächliche Verunreinigungen und durch energieabhängige Resonanzeffekte beeinflußt. Im AlK $\alpha$-Spektrum beobachtet man die Photoemission der 5p-Elektronen im Bereich von $20-28 \mathrm{eV}$, die der 4f-Elektronen bei $8,3 \mathrm{eV}$ und - sehr viel schwächer - die Emission aus dem Leitungsband [11-14]. Wegen der hohen Intensität der 4f-Emission wird diese auch mit der Satellitenlinie $\mathrm{AlK} \alpha_{3,4}$ beobachtet. Im HeII-Spektrum ist die Emission aus dem 4f- und dem Leitungsband etwa gleich intensiv. Die mit $\mathrm{K}$ bezeichnete Struktur wächst mit der Meßzeit an und entspricht daher einer Probenkontamination. Oberhalb der Fermikante tritt eine Satelliten-Struktur (4f mit 50,3 eV angeregt) auf. Das HeISpektrum zeigt dort gleichfalls eine Satelliten-Struktur (Leitungsband mit 23,09 eV). Im Bereich des Leitungsbandes wird eine ausgeprägte Struktur bei $2,8 \mathrm{eV}$ und eine Schulter bei $0,5 \mathrm{eV}$ beobachtet. Möglicherweise han-

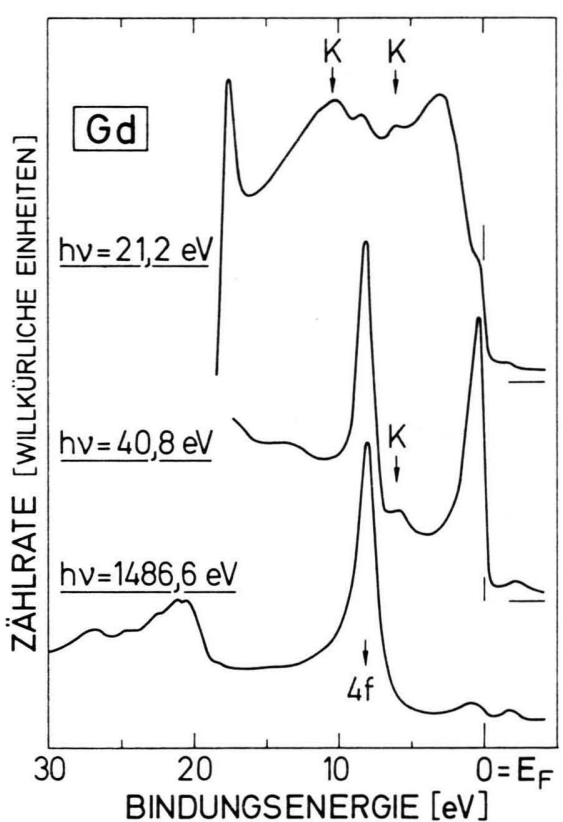

Abb. 1. Photoelektronenspektren von Gadolinium, die mit den angegebenen Anregungsenergien erhalten wurden. Die mit $\mathrm{K}$ bezeichneten Strukturen nehmen mit der Meßzeit $\mathrm{zu}$ und werden auf Kontaminationen zurückgeführt. Die Form der 5p-Struktur im XPS-Spektrum deutet auf Multiplett-Kopplung hin. (Zur Zuordnung der Satelliten-Strukturen oberhalb der Fermi-Kante $E_{\mathrm{F}}$ vgl. Text.) 


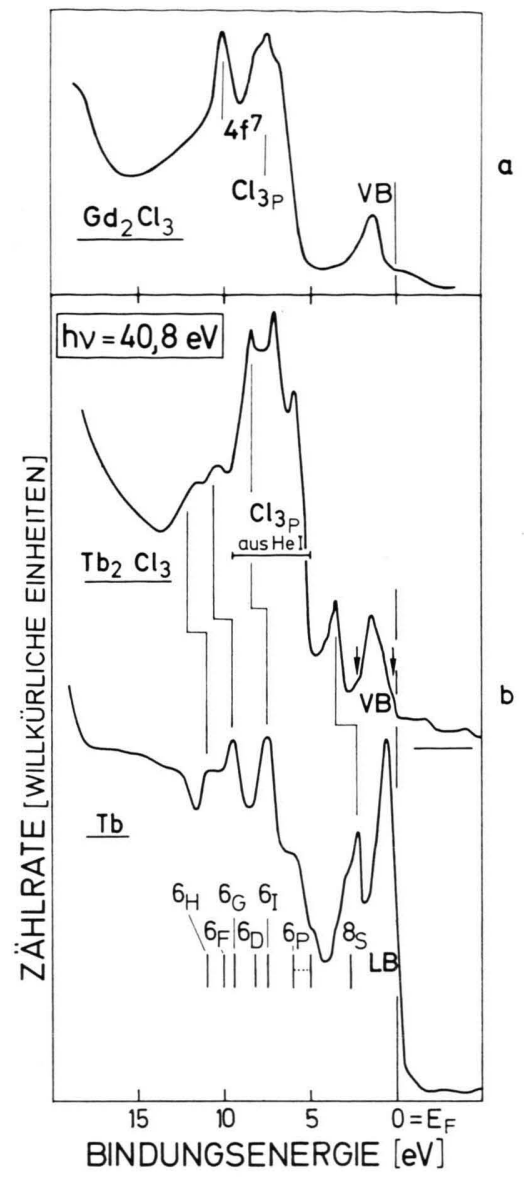

Abb. 2. HeII-Spektren von a) $\mathrm{Gd}_{2} \mathrm{Cl}_{3}$ sowie b) $\mathrm{Tb}_{2} \mathrm{Cl}_{3}$ und Tb. VB und LB bezeichnen das Valenz- bzw. Leitungsband. Die Termwerte des ${ }^{8} \mathrm{f}$-Multipletts sind im Tb-Spektrum angegeben.

delt es sich dabei um die von Keeton und Loucks berechnete [15], bislang experimentell jedoch nicht bestätigte Doppelstruktur des Valenzbandes. Diese Interpretation ist unsicher, weil im HeI-Spektrum die Wiedergabe der elektronischen Zustandsdichte durch Resonanzeffekte verfälscht ist. Außerdem treten stets die mit $\mathrm{K}$ bezeichneten und mit der Zeit stärker werdenden Strukturen auf.

Für die Diskussion der chemischen Bindung in den metallreichen Phasen $\mathrm{Gd}_{2} \mathrm{Cl}_{3}$ und $\mathrm{Tb}_{2} \mathrm{Cl}_{3}$ erscheint daher eine Beschränkung auf die HeIISpektren sinnvoll. Besondere Bedeutung kommt hierbei der Photoemission aus den 4f-Bändern zu: Liegt $\mathrm{Gd}^{3^{+}}$mit einer halbbesetzten $\left(4 \mathrm{f}^{7}-\right)$ Konfiguration vor, so erfolgt die Anregung aus dem Grundzustand ${ }^{8} \mathrm{~S}_{7 / 2}$ nach ${ }^{7} \mathrm{~F}$. Letzterer ist durch Spin-Bahn(LS)Kopplung zwar in 7 Einzelniveaus aufgespalten, die sich jedoch auch bei UV-Anregung nicht auflösen lassen. Für $\mathrm{Gd}^{2+}$ (Anregung $4 \mathrm{f}^{8} \rightarrow 4 \mathrm{f}^{7}$ ) müßte eine Multiplettstruktur auftreten [16]. In diesem Zusammenhang ist $\mathrm{zu}$ betonen, daß die Identifizierung des Cl 3p-Bandes eindeutig aus der Intensitätszunahme im Spektrum von $\mathrm{GdCl}_{3}$ wie auch durch den Vergleich mit HeI-Spektren folgt, in denen die Photoemission wegen des hohen Wirkungsquerschnitts der $\mathrm{Cl} 3 p$-Elektronen von diesen dominiert wird. Das Auftreten einer einzelnen scharfen Struktur für die Photoemission der 4f-Elektronen sowohl bei Gd (Abb. 1) als auch $\mathrm{Gd}_{2} \mathrm{Cl}_{3}$ (Abb. 2 a) beweist das Vorliegen der Konfiguration $4 \mathrm{f}^{7}\left(\mathrm{Gd}^{3+}\right)$. Die in Abb. $2 \mathrm{~b}$ wiedergegebenen HeII-Spektren von $\mathrm{Tb}$ und $\mathrm{Tb}_{2} \mathrm{Cl}_{3}$ zeigen die für eine Anregung aus dem $4 \mathrm{f}^{8}$-Grundzustand $\left(\mathrm{Tb}^{3+}\right)$ erwartete Multiplett-Struktur. Die Interpretation des $\mathrm{Tb}_{2} \mathrm{Cl}_{3}$-Spektrums bereitet wegen der Überlagerung der $\mathrm{Tb} 4 \mathrm{f}-$ und $\mathrm{Cl} 3 \mathrm{p}$-Emission $(5-9,5 \mathrm{eV})$ Schwierigkeiten. Eine Zuordnung fast aller Strukturen ist jedoch möglich, indem zunächst der Betrag der chemischen Verschiebung bei der Verbindungsbildung $(1,3 \mathrm{eV})$ aus der Verschiebung des ${ }^{8} \mathrm{~S}$-Niveaus bestimmt wird. Die auf diese Weise nicht zugeordnete Struktur um $7 \mathrm{eV}$ entspricht dem Maximum der $\mathrm{Cl}$ 3p-Emission, die aus HeISpektren bestimmt wurde; die Struktur bei $6 \mathrm{eV}$ hängt - wie bei $\mathrm{Gd}_{2} \mathrm{Cl}_{3}$ - möglicherweise mit Verunreinigungen der Probe zusammen.

Die vorliegenden Untersuchungen zeigen, daß Gd und $\mathrm{Tb}$ in den Verbindungen $\mathrm{Gd}_{2} \mathrm{Cl}_{3}$ und $\mathrm{Tb}_{2} \mathrm{Cl}_{3}$ die Elektronenkonfiguration $4 \mathrm{f}^{7}$ bzw. $4 \mathrm{f}^{8}$ wie in den freien Metallen und ihren Verbindungen in der Oxidationsstufe +3 haben. In Übereinstimmung mit dem Ergebnis einer Bandstrukturrechnung für $\mathrm{Gd}_{2} \mathrm{Cl}_{3}$ [17] erweisen sich die Lanthanoide in ihrer Chemie der metallreichen Halogenide, speziell in der Ausbildung von Metall-Metall-Bindungen, als dMetalle.

Die Zustandsdichten im Bereich der Fermikante erlauben erste Rückschlüsse auf die elektronischen Transporteigenschaften der Verbindungen $\mathrm{Gd}_{2} \mathrm{Cl}_{3}$ und $\mathrm{Tb}_{2} \mathrm{Cl}_{3}$. Das in Abb. 2 a wiedergegebene Spektrum für $\mathrm{Gd}_{2} \mathrm{Cl}_{3}$ zeigt, daß das Valenzband nur bis etwa $0,5 \mathrm{eV}$ unterhalb des Ferminiveaus besetzt ist. Danach ist die Verbindung ein Halbleiter mit einer Bandlücke von ungefähr $1 \mathrm{eV}$, wenn man annimmt, daß das Ferminiveau in der Mitte der Bandlücke liegt. Dieses zunächst überraschende Ergebnis (s. Einleitung) konnte durch Messungen der elektrischen Leitfähigkeit [18] sowie durch Bandstruktur- 
rechnungen [17] verifiziert werden. Im Gegensatz zum $\mathrm{Gd}_{2} \mathrm{Cl}_{3}$ erscheint im Spektrum von $\mathrm{Tb}_{2} \mathrm{Cl}_{3}$ das Valenzband bis zum Ferminiveau besetzt. Die Deutung im Sinne eines stark p-dotierten Halbleiters steht im Widerspruch zu der experimentell gefundenen Leitfähigkeit [18] der Verbindung. Vielmehr

[1] J. E. Mee u. J. D. Corbett, Inorg. Chem. 4, 88 (1965).

[2] D. A. Lokken u. J. D. Corbett, Inorg. Chem. 12, 556 (1973).

[3] A. Simon, N. Holzer u. Hj. Mattausch, Z. anorg. allg. Chem. 456, 207 (1979).

[4] A. Simon, Hj. Mattausch u. N. Holzer, Angew. Chem. 88, 685 (1976), [I.E. 15, 624 (1976)].

[5] A. Simon, Angew. Chem. 98, 23 (1981), [I.E. 20, 1 (1981)].

[6] Hj. Mattausch, A. Simon, N. Holzer u. R. Eger, Z. anorg. allg. Chem 466, 7 (1980).

[7] S. P. Kowalczyk, Ph. D. Thesis University of California, Berkeley 1976, U.S.A.

[8] B. D. Padalia, W. C. Liang, P. R. Norris, L. W. Watson u. D. J. Fabian, Proc. Roy. Soc. London A 354, 269 (1977).

[9] M. Cardona, C. M. Penchina, N. J. Shevchik u. J. Tejeda, Solid State Commun. 11, 1655 (1972). deuten die Schultern bei 0,3 und $2,3 \mathrm{eV}$ (Pfeile in Abb. 2) auf einen geringen Überschuß an $\mathrm{Tb}$ in der untersuchten Probe hin; d.h. in Abwesenheit von metallischem $\mathrm{Tb}$ sollte $\mathrm{Tb}_{2} \mathrm{Cl}_{3}$ ebenfalls ein Halbleiter mit einer Bandlücke von mindestens $0,3 \mathrm{eV}$ sein.

[10] N. J. Shevchik u. C. M. Penchina, phys. stat. sol. (b) 70, 619 (1975).

[11] F. R. McFeely, S. P. Kowalczik, L. Ley u. D. A. Shirley, Physics Letters 45 A, 227 (1973).

[12] D. E. Eastman, Solid State Commun. 7, 1697 (1969).

[13] Y. Baer u. G. Busch, J. Electr. Spectr. Rel. Phenom. 5, 611 (1974).

[14] M. Campagna, G. K. Wertheim u. Y. Baer, in: Photoemission in Solids (Topics in Applied Physics, Bd. 27, Herausgeber L. Ley und M. Cardona), Springer-Verlag, Berlin 1979, S. $217 \mathrm{ff}$.

[15] S. C. Keeton u. T. L. Loucks, Phys. Rev. 168, 672 (1968).

[16] G. K. Wertheim, A. Rosencwaig, R. L. Cohen u. H. J. Guggenheim, Phys. Rev. Lett. 27, 505 (1971).

[17] D. W. Bullet, Inorg. Chem. 19, 1780 (1980).

[18] W. Bauhofer u. A. Simon, Z. Naturforsch. (im Druck). 